\title{
Journal of Thoracic and Cardiovascular Surgery
}

\section{Editorial Staff}

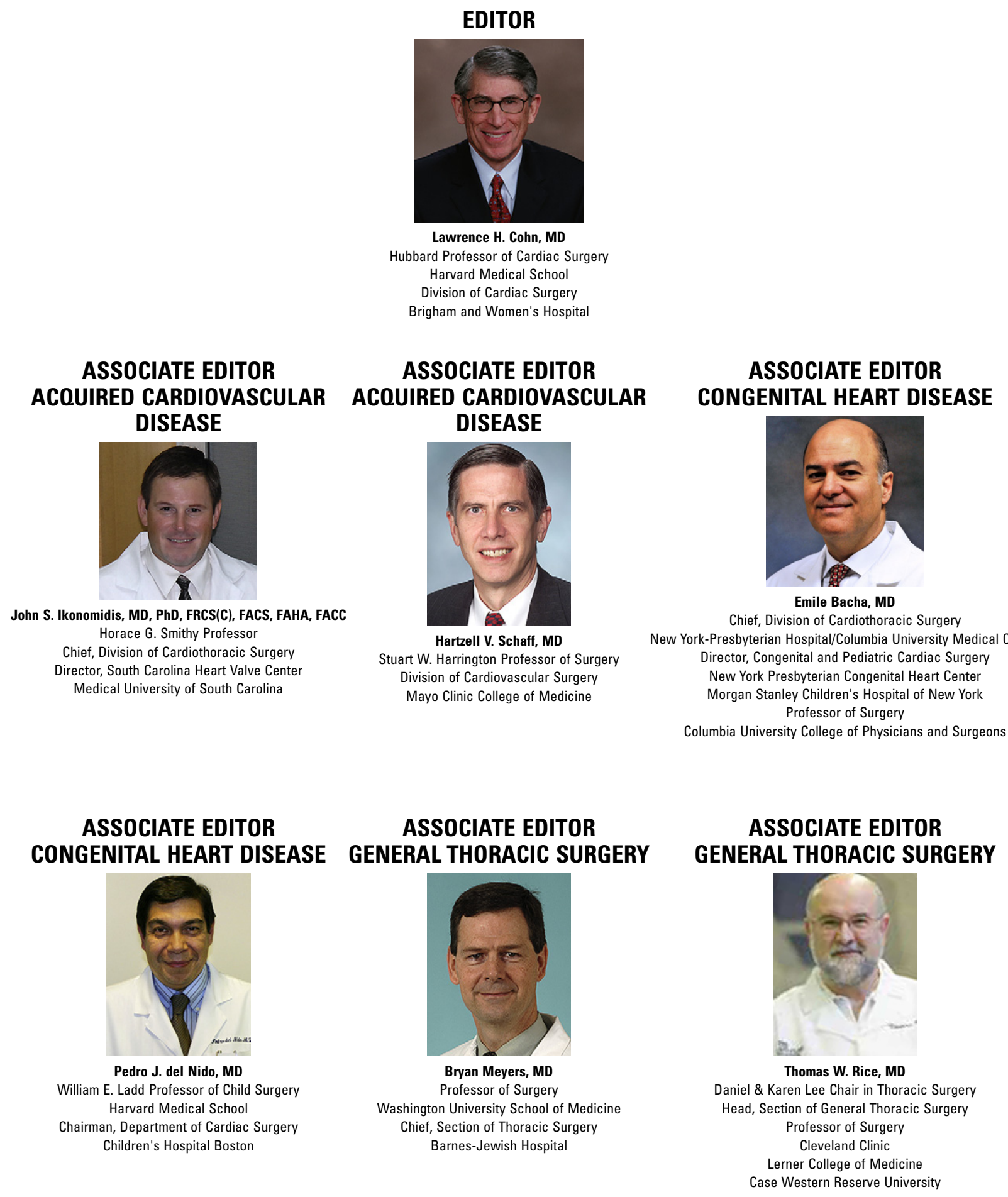




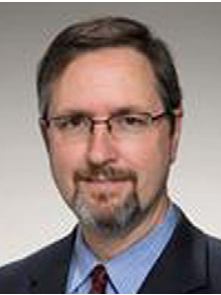

Frank W. Sellke, MD

Karl Karlson and Gloria Karlson Professor of Cardiothoracic Surgery

Chief of Cardiothoracic Surgery

Alpert Medical Schoo

Brown University

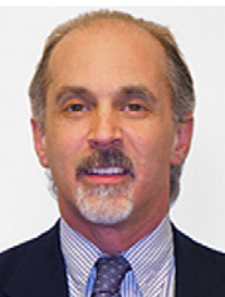

Stanton K. Shernan, MD, FAHA, FASE

Associate Professor of Anesthesia Harvard Medical School Chief, Division of Cardiac Anesthesia Brigham and Women's Hospital

\section{ASSOCIATE EDITOR STATISTICS}

\section{CARDOTHROACIC SURGICAL EDUCATION \& TRAINING}

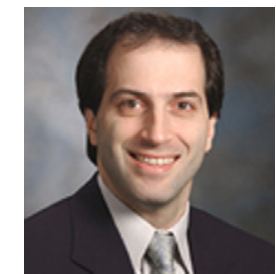

Ara A. Vaporciyan, MD, FACS Professor of Surgery and Deputy Chair

Director of Clinical Education \& Training

Department of Thoracic and Cardiovascular Surger

University of Texas M. D. Anderson Cancer Center

\section{ASSOCIATE EDITOR ETHICS}

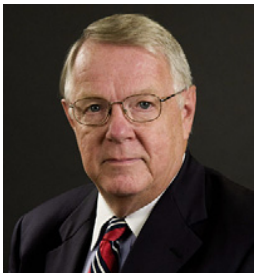

Cary W. Akins, MD Clinical Professor of Surgery Harvard Medical School Massachusetts General Hospital

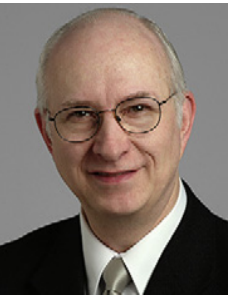

Eugene H. Blackstone, MD Director, Clinical Investigations Heart and Vascular Institute Cleveland Clinic
ASSOCIATE EDITOR CHINESE EDITION

Shengshou Hu, MD, FACC

Director, National Center for Cardiovascular Diseases of China President, Fuwai Hospital

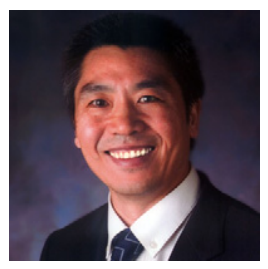

\section{MANAGING EDITOR}

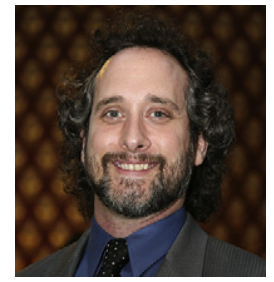

Ryan Walther, $M A$

AATS Scientific Publications

American Assocation for Thoracic Surgery 


\section{AATS Officers and Councilors}

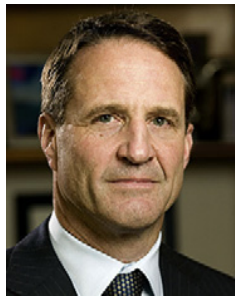

President

Craig R. Smith

Columbia University

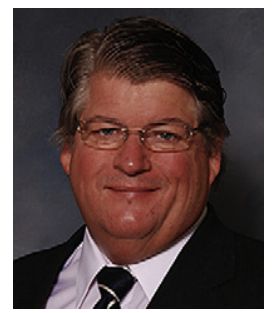

Vice President David J. Sugarbaker Brigham and Women's Hospital

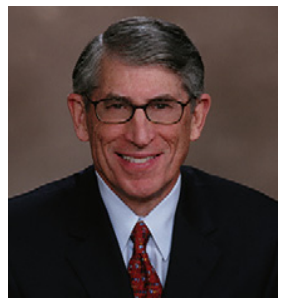

Editor

Lawrence H. Cohn

Brigham and Women's Hospital

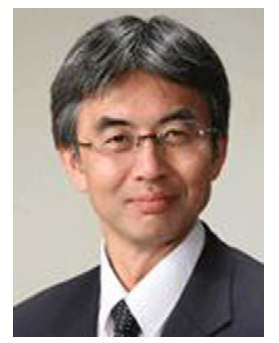

Councilor

Hiroshi Date

Kyoto University

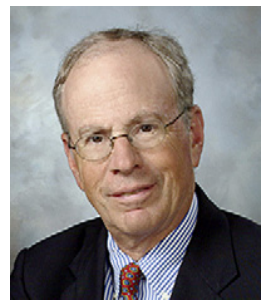

Councilor

Irving L. Kron

University of Virginia

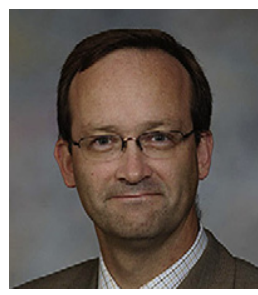

Secretary

Thoralf M. Sundt III

Massachusetts General Hospital

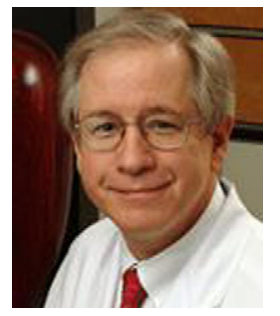

Councilor

Joseph S. Coselli

Baylor College of Medicine

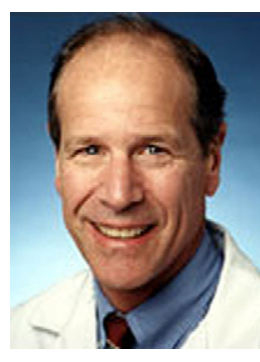

Councilor

Bartley P. Griffith

University of Maryland

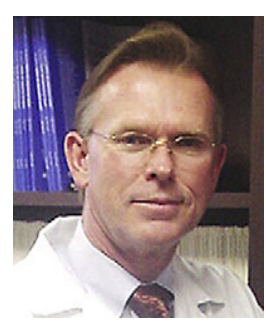

Councilor

Vaughn A. Starnes

University of Southern California

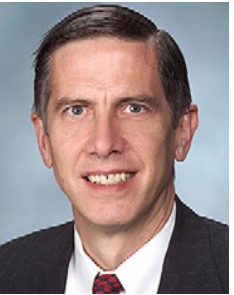

President-Elect Hartzell V. Schaff

Mayo Clinic

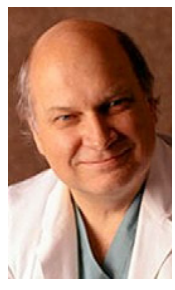

Treasurer

Duke Cameron

The Johns Hopkins Hospital

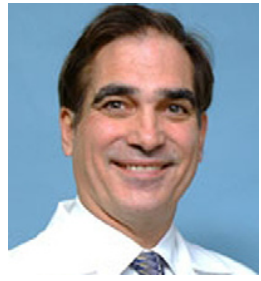

Councilor

Ralph J. Damiano, Jr.

Washington University

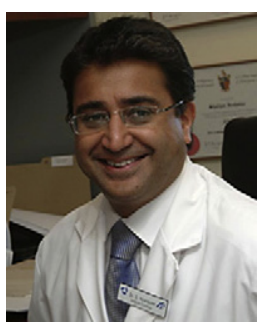

Councilor

Shaf Keshavjee

Toronto General Hospital

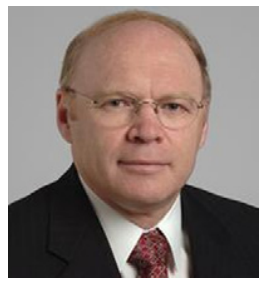

Councilor

Lars G. Svensson

Cleveland Clinic 